Series A

I. MATHEMATICA

477

\title{
SCHWARZIAN DERIVATIVES \\ AND HOMEOMORPHIC EXTENSIONS
}

BY

P. L. DUREN and O. LEHTO

H E L S I K I 1970

S U O M A L A I E N TIEDEAKATEM I A

doi:10.5186/aasfm.1971.477 
Communicated 10 October 1969.

KESKUSKIRJAPAINOO

HELSINKI 1970 


\section{Introduction}

Some years ago, Nehari [7] found a remarkable connection between the Schwarzian derivative

$$
\{w,\}=\left(\frac{w^{\prime \prime}}{w^{\prime}}\right)^{\prime}-\frac{1}{2}\left(\frac{w^{\prime \prime}}{w^{\prime}}\right)^{2}
$$

and univalence. He showed that if $f$ is analytic in the unit disk and

$$
|\{f, z\}| \leq 2\left(1-|z|^{2}\right)^{-2}, \quad|z|<1,
$$

then $f$ is univalent. Hille [3] showed that the constant 2 is best possible; that is, for no $k>2$ does the condition

$$
|\{f, z\}| \leq k\left(1-|z|^{2}\right)^{-2}, \quad|z|<1,
$$

imply univalence. On the other hand, every univalent function satisfies (2) with $k=6$, as Nehari showed, and this inequality is sharp for each fixed $z$.

More recently, Ahlfors and Weill [2] proved that if an analytic function $f$ satisfies (2) for some constant $k<2$, then $f$ has a quasiconformal extension to the entire complex plane. In particular, $f$ maps the unit disk onto a Jordan domain; that is, onto the interior of a Jordan curve on the Riemann sphere. The purpose of the present paper is to show that the theorem of Ahlfors and Weill remains essentially true when the constant $k<2$ is replaced by a function of $|z|$ which increases "slowly" to 2 as $|z| \rightarrow 1$. Specifically, our main result is as follows.

Theorem. Let $\lambda$ be nondecreasing on the interval $0 \leq r<1$, with $0<\lambda(r)<1$ and

$$
\frac{1}{1-\lambda(r)}=O\left(\log \frac{1}{1-r}\right), \quad r \rightarrow 1 .
$$

Let $f$ be analytic in $|z|<1$, and let its Schwarzian derivative satisfy

$$
|\{f, z\}| \leq \frac{2 \lambda(|z|)}{\left(1-|z|^{2}\right)^{2}}, \quad|z|<1 .
$$

Then $f$ is univalent in $|z|<1$ and has a homeomorphic extension to the whole plane. The continuation is quasiconformal with the possible exception of the unit circle $|z|=1$. 
Our proof involves no estimates of solutions to differential equations, and it makes no appeal to Nehari's theorem. We first construct a normal family of quasiconformal mappings which are approximate extensions of $f$ (cf. Ahlfors [1]). The family is proved to be normal, and the desired homeomorphic extension is obtained as a limit.

The growth condition (3) can be relaxed, as we point out at the end of the paper.

\section{Approximate quasiconformal extensions}

The first step in the proof is to construct a certain quasiconformal mapping of the extended plane onto itself which agrees with the function $f$ in a given disk $|z| \leq r<1$.

Let $S(z)=\{f, z\}$, and let $w_{1}$ and $w_{2}$ be linearly independent solutions to the differential equation

$$
w^{\prime \prime}+\frac{1}{2} S w=0
$$

normalized so that $w_{1}(0)=w_{2}^{\prime}(0)=0$ and $w_{1}^{\prime}(0)=w_{2}(0)=1$. Thus

$$
w_{1}^{\prime}(z) w_{2}(z)-w_{1}(z) w_{2}^{\prime}(z)=1
$$

for every $z$ in the unit disk. The most general analytic function $g$ with Schwarzian derivative $S$ then has the form $g=T \circ\left(w_{1} / w_{2}\right)$, where $T$ is an arbitrary linear fractional mapping. Hence we may assume $f=w_{1} / w_{2}$.

The functions $w_{1}$ and $w_{2}$ are analytic in $|z|<1$ and cannot vanish simultaneously, by (5). This shows that $w_{2}$ has no zeros in $|z|<1$, since $w_{1} / w_{2}$ is analytic. Thus $\left.f^{\prime}=\left[w_{2}\right)\right]^{-2}$ is finite and nonvanishing in $|z|<1$. In particular, $f$ is locally conformal in the open unit disk.

Now fix $r, 0<r<1$. In $|z|>r^{2}$, define the function $g=\varphi_{1} / \varphi_{2}$, where

$$
\varphi_{i}(z)=w_{i}(\zeta)+(z-\zeta) w_{i}^{\prime}(\zeta), \quad i=1,2,
$$

and $\zeta=r^{2} / \bar{z}$. It follows from (5) that $\varphi_{1}$ and $\varphi_{2}$ cannot vanish simultaneously. The function $g$ has continuous first partial derivatives except at the zeros (if any) of $\varphi_{2}$. In view of the relations

$$
w_{1}^{\prime \prime} w_{2}-w_{1} w_{2}^{\prime \prime}=0
$$

and

$$
w_{1}^{\prime \prime} w_{2}^{\prime}-w_{1}^{\prime} w_{2}^{\prime \prime}=\frac{1}{2} S
$$


a calculation gives

$$
g_{z}(z)=\left[\varphi_{2}(z)\right]^{-2}
$$

and

$$
g_{\bar{z}}(z)=-\frac{1}{2}\left[\varphi_{2}(z)\right]^{-2}(r / \bar{z})^{2}(z-\zeta)^{2} S(\zeta),
$$

where $g_{z}=\frac{1}{2}\left(g_{x}-i g_{y}\right)$ and $g_{\bar{z}}=\frac{1}{2}\left(g_{x}+i g_{y}\right), z=x+i y$. Let $\mu=g_{\bar{z}} / g_{z}$ denote the complex dilatation of $g$. Then we have

$$
\begin{aligned}
\mu(z) & =-\frac{1}{2}(r / \bar{z})^{2}(z-\zeta)^{2} S(\zeta) \\
& =-\frac{1}{2}(\zeta / \bar{\zeta})^{2} r^{-2}\left(r^{2}-|\zeta|^{2}\right)^{2} S(\zeta)
\end{aligned}
$$

Since $0 \leq r^{-1}\left(r^{2}-|\zeta|^{2}\right)<1-|\zeta|^{2}$, it follows from (4) that

$$
|\mu(z)| \leq \lambda(|\zeta|)<1, \quad|z|>r^{2}
$$

For the Jacobian $J=\left|g_{z}\right|^{2}-\left|g_{\bar{z}}\right|^{2}$ we have

$$
J(z)=\left|\varphi_{2}(z)\right|^{-4}\left(1-|\mu(z)|^{2}\right), \quad|z|>r^{2} .
$$

Thus the function $g$ is locally homeomorphic in $|z|>r^{2}$, except perhaps at the zeros of $\varphi_{2}$. But $g$ is locally homeomorphic at these points also, since we can repeat the above calculations for the function $1 / g$. Hence $g$ is locally a quasiconformal mapping in $|z|>r^{2}$.

Now define the function

$$
F(z)= \begin{cases}f(z), & |z| \leq r \\ g(z), & |z|>r .\end{cases}
$$

By the definition of $g$ it is clear that $F$ is continuous (with respect to the spherical metric) in the whole extended plane. Since $f$ is locally conformal in $|z|<1$ and $g$ is locally quasiconformal in $|z|>r^{2}$, the function $F$ has generalized $L^{2}$-derivatives (see [6]). Consequently, $F$ is a generalized $L^{2}$-solution to the Beltrami equation $w_{\bar{z}}=\mu w_{z}$, where $\mu(z)$ is defined as above for $|z|>r$ and $\mu(z)=0$ for $|z|<r$. From this it follows ([6]) that $F$ admits a representation

$$
F=q \circ \psi,
$$

where $\psi$ is a quasiconformal homeomorphism of the extended plane onto itself and $q$ is a rational function.

We have observed that $F$ is locally homeomorphic in $|z|<r$ and in $|z|>r$. In order to show that it is locally homeomorphic on $|z|=r$ 
also, we introduce the following lemma. Recall that an interior function is a continuous light open mapping ([6]).

Lemma 1. Let $C$ be an analytic arc passing through a point $z_{0}$ and dividing a disk $D$ centered at $z_{0}$ into the domains $D_{1}$ and $D_{2}$. Let $F$ be an interior function in $D$ and let $f_{i}$ be the restriction of $F$ to $D_{i}$, $i=1,2$. If $f_{1}$ is injective in a neighborhood of $z_{0}$ and

$$
f_{2}(z)=a+b\left(z-z_{0}\right)+o\left(\left|z-z_{0}\right|\right), \quad b \neq 0,
$$

as $z \rightarrow z_{0}$, then $F$ is homeomorphic at $z_{0}$.

Proof. There is no loss of generality in supposing that $C$ is a segment of the real axis and that $z_{0}=0, a=0$, and $b=1$. In view of Stoilow's theorem, there is a neighborhood $V \subset D$ of $z=0$ in which $F$ has the form

$$
F=h^{n},
$$

where $h$ is a homeomorphism and $n$ is a positive integer. By hypothesis, we may suppose that the restriction of $F$ to $V \cap D_{1}$ is an injection, and that

$$
\left|f_{2}(z)-z\right|<|z|, \quad z \in V \cap D_{2} .
$$

In particular, $\operatorname{Im}\{F(z)\}<0$ for $z \in V \cap D_{2}$. Hence $n=1$ in (9), and the restriction of $F$ to $V$ is a homeomorphism.

Remark. The assumption (8) is essential, as the counterexample $f_{1}(z)=z, f_{2}(z)=z^{2} / \bar{z}$ shows, with $C$ a segment $-\varrho \leq z \leq \varrho$.

If we apply the above lemma with $f_{1}=g, f_{2}=f$, we conclude that the function $F$ given by (7) is locally homeomorphic on $|z|=r$. Hence, in (7), the rational function $q$ has no branch points in the finite plane. It follows that $q$ is a linear fractional transformation. Thus $F$ is a quasiconformal homeomorphism of the extended plane onto itself.

\section{Equicontinuous families of mappings}

Now choose an arbitrary sequence $\left\{r_{n}\right\}$ with $0<r_{n}<r_{n+1}<1$ and $r_{n} \rightarrow 1$ as $n \rightarrow \infty$. Let $F_{n}$ be the quasiconformal mapping just constructed, corresponding to $r=r_{n}$, and let $\mu_{n}$ be the complex dilatation of $F_{n}$. Then $\mu_{n}(z)=0$ for $|z|<r_{n}$ and by (6),

$$
\left|\mu_{n}(z)\right| \leq \lambda\left(r_{n}^{2} /|z|\right), \quad|z|>r_{n} .
$$

For each fixed $z_{0}$ and for $\varrho>0$, let

$$
M_{n}\left(\varrho, z_{0}\right)=\int_{0}^{2 \pi} \frac{d \vartheta}{1-\left|\mu_{n}\left(z_{0}+\varrho e^{i \vartheta}\right)\right|} \cdot
$$


We shall make use of the following lemma.

Lemma 2. If $\lambda$ satisfies the condition (3), then for each $z_{0}$,

$$
\int_{\varrho_{2}}^{\varrho_{2}} \frac{d \varrho}{\varrho M_{n}\left(\varrho, z_{0}\right)} \geq \alpha\left(\varrho_{1}, \varrho_{2}, z_{0}\right), \quad 0<\varrho_{1}<\varrho_{2}<\infty,
$$

where $\alpha$ is independent of $n, \alpha\left(\varrho_{1}, \varrho_{2}, z_{0}\right)>0$ for $\varrho_{1}<\varrho_{2}$, and $\alpha\left(\varrho_{1}, \varrho_{2}, z_{0}\right) \rightarrow \infty$ if $\varrho_{2}$ is fixed and $\varrho_{1} \rightarrow 0$, or if $\varrho_{1}$ is fixed and $\varrho_{2} \rightarrow \infty$.

Postponing the proof, let us show how Lemma 2 can be used to complete the proof of the main theorem. If the sequence $\left\{F_{n}\right\}$ has the property (11) given by the lemma, then as proved in [4] (see also [5]), $\left\{F_{n}\right\}$ is equicontinuous with respect to the spherical metric at each point of the extended complex plane $\Omega$. It therefore follows from Ascoli's theorem that a subsequence converges uniformly in $\Omega$ to a continuous function $G$. But it is also shown in [4] that under the condition (11), the limit function $G$ must be a homeomorphism of $\Omega$ onto itself. Furthermore, $G$ satisfies the Beltrami equation $w_{\bar{z}}=\nu w_{z}$ in $|z|>1$, where

$$
v(z)=\lim _{n \rightarrow \infty} \mu_{n}(z)=-\frac{1}{2}(\bar{z} / z)^{2}\left(1-|z|^{-2}\right)^{2} S(1 / \bar{z})
$$

for $|z|>1$. Thus, by (4),

$$
|v(z)| \leq \lambda(1 /|z|)<1, \quad|z|>1,
$$

which shows that $G$ is locally quasiconformal in $|z|>1$. Of course, it is clear from the construction of $F_{n}$ that $G(z)=f(z)$ for $|z|<1$. Hence $G$ is the desired homeomorphic extension of $f$. In particular, $f$ is univalent in $|z|<1$. This proves the theorem.

\section{Proof of Lemma 2}

The proof of Lemma 2 rests upon the following lemma.

Lemma 3. If $\lambda$ satisfies the condition (3), then for each $z_{0}$,

$$
M_{n}\left(\varrho, z_{0}\right)=O(\log 1 / \varrho), \quad \varrho \rightarrow 0,
$$

uniformly in $n$.

Proof of Lemma 3. Suppose first that $\left|z_{0}\right|<1$, and choose $N$ such that $r_{N} \leq\left|z_{0}\right|<r_{N+1}$. Then for $n=1,2, \ldots, N$, we have by (10)

$$
\left|\mu_{n}(z)\right| \leq \lambda\left(r_{n}\right) \leq \lambda\left(\left|z_{0}\right|\right), \quad|z|>r_{n},
$$

while $\mu_{n}(z)=0$ for $|z|<r_{n}$. For $n \geq N+1$, we have $\mu_{n}(z)=0$ for $|z|<r_{N+1}$. Thus $M_{n}\left(\varrho, z_{0}\right)$ remains bounded, uniformly in $n$, as $\varrho \rightarrow 0$. 
Next suppose $\left|z_{0}\right|>1$. Then

$$
\left|\mu_{n}(z)\right| \leq \lambda(1 /|z|) \leq \lambda\left(2 /\left(1+\left|z_{0}\right|\right)\right)<1
$$

if $\left|z-z_{0}\right| \leq \frac{1}{2}\left(\left|z_{0}\right|-1\right)$. This again shows that $M_{n}\left(\varrho, z_{0}\right)$ is uniformly bounded as $\varrho \rightarrow 0$.

Finally, suppose $\left|z_{0}\right|=1$. Then in view of the rotational symmetry, we may assume $z_{0}=1$. Fix $\varrho, 0<\varrho<1$. If $0<r_{n}<1-\varrho$, we have by (10) and (3)

$$
M_{n}(\varrho, 1) \leq \frac{2 \pi}{1-\lambda(1-\varrho)} \leq C \log 1 / \varrho .
$$

On the other hand, if $1-\varrho \leq r_{n}<1$, we have by (10)

$$
M_{n}(\varrho, 1) \leq 2 \int_{0}^{\beta_{n}} \frac{d \vartheta}{1-\lambda\left(r_{n}^{2} /\left|1+\varrho e^{i, \mid}\right|\right)}+\pi,
$$

where $\left|1+\varrho e^{i \beta_{n}}\right|=r_{n}$ and $\pi / 2<\beta_{n} \leq \pi$. If we set

$$
x=\left|1+\varrho e^{i \vartheta}\right| \text { and } u=\cos \vartheta=\left\{x^{2}-\left(1+\varrho^{2}\right)\right\} / 2 \varrho,
$$

the integral in (13) transforms to

$$
\int_{\gamma_{n}}^{1} \frac{d u}{\sqrt{1-u^{2}}\left\{1-\lambda\left(r_{n}^{2} / x\right)\right\}}=\int_{\gamma_{n}}^{0}+\int_{0}^{1}=I_{1}+I_{2},
$$

say, where

$$
\gamma_{n}=\left\{r_{n}^{2}-\left(1+\varrho^{2}\right)\right\} / 2 \varrho .
$$

But

$$
\begin{aligned}
I_{2} & \leq\left\{1-\lambda\left(1 / \sqrt{1+\varrho^{2}}\right)\right\}^{-1} \int_{0}^{1} \frac{d u}{\sqrt{1-u^{2}}} \\
& \leq \frac{\pi}{2}\left\{1-\lambda\left(1-\frac{1}{4} \varrho^{2}\right)\right\}^{-1} \leq C \log 1 / \varrho,
\end{aligned}
$$

by (3) and the inequality

$$
1 / \sqrt{1+\varrho^{2}} \leq 1-\frac{1}{4} \varrho^{2}, \quad 0<\varrho<1 .
$$

It remains now to estimate $I_{1}$. If $1-\varrho \leq r_{n}<\sqrt{1-\varrho^{2}}$, we have 


$$
\begin{aligned}
I_{1} \leq\left\{1-\lambda\left(r_{n}\right)\right\}^{-1} \int_{-1}^{0} \frac{d u}{\sqrt{1-u^{2}}} \leq \frac{\pi}{2}\left\{1-\lambda\left(\sqrt{1-\varrho^{2}}\right)\right\}^{-1} \\
\leq \frac{\pi}{2}\left\{1-\lambda\left(1-\frac{1}{2} \varrho^{2}\right)\right\}^{-1} \leq C \log 1 / \varrho .
\end{aligned}
$$

On the other hand, if $\sqrt{1-\varrho^{2}} \leq r_{n}<1$, we find

$$
I_{1} \leq \frac{1}{\varrho \sqrt{1-\gamma_{n}^{2}}} \int_{r_{n}}^{\sqrt{1+\varrho^{2}}} \frac{x d x}{1-\lambda\left(r_{n}^{2} x\right)} .
$$

Setting $t=r_{n}^{2} / x$, observing that $\gamma_{n}^{2} \leq \varrho^{2}$, and assuming $\varrho^{2} \leq \frac{1}{2}$, we therefore find

$$
I_{1} \leq \frac{3}{\varrho} \int_{a_{Q}}^{1} \frac{d t}{1-\lambda(t)}
$$

where

$$
a_{\varrho}=\left(1-\varrho^{2}\right) / \sqrt{1+\varrho^{2}} \geq 1-2 \varrho^{2}, \quad \varrho^{2} \leq \frac{1}{2} .
$$

Thus in view of (3), we have for $\varrho^{2} \leq \frac{1}{4}$

$$
I_{1} \leq \frac{C}{\varrho} \int_{1-2 \varrho^{2}}^{1} \log \left(\frac{1}{1-t}\right) d t=O(\varrho \log 1 / \varrho) .
$$

This completes the proof of Lemma 3 .

Proof of Lemma 2. It follows from Lemma 3 that the integral (11) is estimated from below by

$$
C \int_{\varrho_{1}}^{\varrho_{2}} \frac{d \varrho}{\varrho \log 1 / \varrho}, \quad 0<\varrho_{1}<\varrho_{2}<1,
$$

which tends to $\infty$ as $\varrho_{1} \rightarrow 0$. On the other hand, for each fixed $z_{0}$, we have

$$
\left|\mu_{n}\left(z_{0}+\varrho e^{i \vartheta}\right)\right| \leq \lambda\left(\frac{1}{2}\right)<1
$$

for all $\varrho \geq R=\left|z_{0}\right|+2$. Thus for $R \leq \varrho_{1}<\varrho_{2}<\infty$, the integral (11) is not less than 


$$
\frac{1}{2 \pi}\left\{1-\lambda\left(\frac{1}{2}\right)\right\} \log \left(\varrho_{2} / R\right),
$$

which tends to $\infty$ as $\varrho_{2} \rightarrow \infty$. This establishes Lemma 2 and hence completes the proof of the main theorem.

\section{Generalizations}

It is clear from the proof of Lemma 2 that the theorem will remain true with a growth restriction on $\lambda$ weaker than (3). What is needed is a condition of the form

$$
\frac{1}{1-\lambda(r)}=O(\psi(r)), \quad r \rightarrow 1,
$$

where $\psi$ is a positive nondecreasing integrable function such that

$$
\int_{0}^{1} \frac{d \varrho}{\varrho \psi(1-\varrho)}=\infty
$$

and such that

$$
\Psi(x)=\int_{1-x}^{1} \psi(t) d t
$$

has the property

$$
\int_{0}^{1} \frac{d \varrho}{\Psi\left(\varrho^{2}\right)}=\infty .
$$

The condition (16) will hold if, for example,

$$
\psi(r)=O\left((1-r)^{-1 / 2}\right),
$$

although such a condition does not imply (15).

The question of the best possible growth condition (14) remains open. It is of interest to find examples showing that the theorem fails without some condition of this form. 


\section{References}

[1] Ahlfors, L., Lectures on Quasiconformal Mappings (Van Nostrand, Princeton, N. J., 1966).

[2] Ahlfors, L. and G. Weill, "A uniqueness theorem for Beltrami equations", Proc. Amer. Math. Soc. 13(1962), 975-978.

[3] Hille, E., "Remarks on a paper of Zeev Nehari», Bull. Amer. Math. Soc. 55(1949), $552-553$.

[4] Leнto, O., "Homeomorphisms with a given dilatation", Proceedings of the 15th Scandinavian Congress, Oslo 1968 (Lecture Notes in Mathematics, vol. 118, Springer-Verlag, Berlin, 1970).

[5] --" - "Remarks on generalized Beltrami equations and conformal mappings», to appear in Proceedings of the Romanian-Finnish Seminar on Teichmüller spaces and quasiconformal mappings, Braşov, 1969.

[6] - - - and K. I. VIrtanen, Quasikonforme Abbildungen (Springer-Verlag, Berlin, 1965).

[7] Nemari, Z., "The Schwarzian derivative and schlicht functions", Bull. Amer. Math. Soc. 55(1949), 545-551.

University of Michigan and

University of Helsinki 